\title{
Diabetes Mellitus, Acute Hyperglycemia, and Ischemic Stroke
}

\author{
Askiel Bruno, MD, MS ${ }^{*}$ \\ David Liebeskind, $M D^{1}$ \\ Qing Hao, MD, PhD ${ }^{1}$ \\ Radoslav Raychev, $M D^{1}$ for the UCLA Stroke Investigators
}

\author{
Address \\ *Department of Neurology, Medical College of Georgia, \\ 1120 15th Street, BI3076, Augusta, GA 30912, USA \\ Email: abruno@mcg.edu \\ ${ }^{1}$ Department of Neurology, University of California Los Angeles \\ School of Medicine, Los Angeles, CA, USA \\ Published online: 21 August 2010 \\ (C) The Author(s) 2010. This article is published with open access at Springerlink.com
}

\section{Opinion statement}

Acute brain ischemia is a dynamic process susceptible to multiple modulating factors, such as blood glucose level. During acute ischemic brain injury, hyperglycemia exacerbates multiple deleterious derangements. Timely and sufficient correction of hyperglycemia during acute brain ischemia may limit the brain injury and improve clinical outcomes. The clinical efficacy of such intervention remains to be proven. Although results from animal and clinical observational studies suggest that hyperglycemia during acute brain ischemia may exacerbate the brain injury, there is no evidence from randomized treatment trials that rapid correction of the hyperglycemia improves outcomes. Given the excess effort, cost, and risk involved in rapid and safe correction of hyperglycemia during acute stroke, less aggressive treatments with subcutaneous insulin seem appropriate at this time. Subcutaneous insulin protocols can maintain blood glucose levels below $200 \mathrm{mg} / \mathrm{dL}$ a majority of the time in most patients, especially if basal insulin is added. When available, an endocrinology consultant can optimize the acute treatment and help the transition to long-term care. Given the multiple reports linking admission hyperglycemia with symptomatic hemorrhagic conversion of ischemic stroke treated with thrombolytic drugs, it may be best to rapidly lower severe hyperglycemia in such patients. For example, if the admission blood glucose is approximately $300 \mathrm{mg} / \mathrm{dL}$ and the patient is a candidate for thrombolytic therapy, consider giving an intravenous bolus of regular insulin 8 units. Somewhat lower or higher insulin doses may be best for lesser or greater hyperglycemia. Such a bolus will start lowering the blood glucose in about 5 min. A temporary continuous intravenous insulin infusion may then be used in most patients to maintain the glucose closer to normal levels (eg, below 180 or $140 \mathrm{mg} / \mathrm{dL}$ ). 


\section{Introduction}

Blood glucose regulation involves intestinal absorption, release into the bloodstream from glycogen stores (glycogenolysis), synthesis (gluconeogenesis) and release into the bloodstream, transport from blood into interstitial spaces and cells throughout the body, and urinary excretion. Levels of blood glucose fluctuate more rapidly and to a greater extent than levels of any other metabolite. Such glucose fluctuations are greater in patients with insulin resistance and during acute illness. Either excessively low or high blood glucose levels have well-known complications. This review focuses on hyperglycemia.

Although the complications of chronic hyperglycemia and acute hyperosmolar severe hyperglycemia are well known, the complications of acute (stress) moderate hyperglycemia are not. Approximately $40 \%$ of patients with acute ischemic stroke have admission hyperglycemia $(>130 \mathrm{mg} / \mathrm{dL})[1,2]$, and the majority of such patients have diabetes mellitus. The hyperglycemia during acute stroke reflects both a spectrum of acute stress and a spectrum of insulin resistance. Our ability to correct glucose deviations rapidly may have important clinical implications in patients with acute ischemic stroke. Here we summarize the data about hyperglycemia during acute ischemic stroke and how it relates to outcomes. We also offer suggestions for treating hyperglycemia during acute stroke.

\section{Diabetes and risk of stroke}

Diabetes mellitus, through chronic hyperglycemia, has been linked with accelerated development of both microvascular disease and atherosclerosis throughout the body. Consequently, diabetes mellitus increases the combined risk of stroke and myocardial infarction by about 2.5 times [3] and the risk of stroke alone 1.7 to 2.1 times [4,5], compared with otherwise similar patients without diabetes. Diabetes also increases the risk of stroke after a transient ischemic attack (TIA), as it represents one of the Ds in the $\mathrm{ABCD}^{2}$ score, which includes age ( 0 or 1$)$, blood pressure level ( 0 or 1$)$, clinical stroke symptoms $(0,1$, or 2$)$, duration of the TIA symptoms $(0,1$, or 2$)$, and diabetes ( 0 or 1$)$ [6]. Risk of stroke increases with increasing $\mathrm{ABCD}^{2}$ score, within the range from 0 to 7 .

Thus it is reasonable to expect that better control of chronic hyperglycemia may reduce the incidence of vascular events. This hypothesis was tested in five randomized clinical trials totaling 33,040 patients: strict glycemic control lowered the hemoglobin A1C by $0.9 \%$ (from $7.5 \%$ to $6.6 \%$ ) compared with standard therapy [7]. In these five clinical trials, strict glycemic control lowered the rate of coronary events, but not stroke or death. A reason for these findings is not readily apparent.

Elevated fasting glucose or diabetes mellitus in combination with other, interrelated vascular risk factors has been defined as the metabolic syndrome [8]. Because of the coexistence of multiple vascular risk factors, patients with the metabolic syndrome have a higher risk for vascular outcomes than those with a single risk factor. The International Diabetes Federation has defined the metabolic syndrome as central obesity plus two of the following four additional derangements: 1) elevated triglycerides, 2) decreased HDL cholesterol, 3) elevated blood pressure, and 4) elevated fasting plasma glucose. Thus, although all patients with the metabolic syndrome have central obesity, they are somewhat heterogeneous with respect to the other risk factors. Consequently, treatment for the metabolic syndrome is individualized and not 
specific to the syndrome. It appears that each risk factor within the metabolic syndrome should be identified and treated as indicated, and that classification into this syndrome offers no clear advantage.

\section{Can animal stroke hyperglycemia studies lead to optimized clinical trials?}

Although animal studies usually precede clinical trials, there is a risk that positive findings in animals will not translate to similar clinical results [9]. In most animal studies, acute hyperglycemia immediately before or during ischemia exacerbates the ischemic brain injury [10-13]. Compared with normoglycemic animals, animals with ischemic stroke and hyperglycemia tend to have more brain edema, hemorrhagic transformation of infarcts, brain herniation, and death. However, not all animal studies confirm such observations. The exacerbated damage with hyperglycemia is usually seen in animal ischemic stroke models with reperfusion and occurs less with permanent occlusion $[12,14]$. This finding suggests that reperfusion of ischemic brain may be an important factor leading to increased brain damage by hyperglycemia [13].

Relatively few animal interventional studies have looked at the effects of hyperglycemia correction in focal brain ischemia [11,15-17]. Some animal interventional studies show a benefit related to reduction of the acute hyperglycemia. Unfortunately, many of the animal studies do not seem clinically relevant because the models involved global and not focal brain ischemia, the glucose levels were unusually high, or the hyperglycemia was corrected around the time that the ischemia began. In some studies using insulin, the animals became severely hypoglycemic $(<45 \mathrm{mg} / \mathrm{dL})$, had seizures, and died. One such study reported a $U$-shaped curve indicating optimal outcome at a glucose level of about $126 \mathrm{mg} / \mathrm{dL}$ and worsening outcomes below and above this level [17].

In an acute ischemic stroke interventional study in rats, one group of 10 rats was made hyperglycemic ( $555 \mathrm{mg} / \mathrm{dL})$ for $2 \mathrm{~h}$ before and $2 \mathrm{~h}$ after permanent middle cerebral artery (MCA) occlusion, another group of ten was made diabetic (blood glucose, $542 \mathrm{mg} / \mathrm{dL}$ ) 4 days before the MCA occlusion and was not treated, another group of ten was made diabetic and was treated with insulin since 2 days before the MCA occlusion (blood glucose, $176 \mathrm{mg} /$ $\mathrm{dL}$ ), and 15 rats were used as controls (blood glucose, 156-176 mg/dL) [15]. Infarct volumes were larger with acute hyperglycemia $\left(161 \mathrm{~mm}^{3}\right)$ and with untreated diabetes $\left(190 \mathrm{~mm}^{3}\right)$ than with insulin-treated diabetes $\left(131 \mathrm{~mm}^{3}\right)$ or in controls $\left(121 \mathrm{~mm}^{3}\right)$. However, a therapeutic effect cannot be translated to clinical acute stroke, as insulin treatment was started 2 days before the MCA occlusion, and blood glucose levels at the time of MCA occlusion were similar to those in the control group.

Unfortunately, the animal studies so far have not established a clear relation between the degree of hyperglycemia and extent of acute ischemic brain injury. Is the relationship linear, as suggested by some clinical studies? Also, a therapeutic time window for hyperglycemia correction has not been established. Is it crucial (as with thrombolytic therapies) to inter- 
vene as early as possible for best results? If so, how far does this therapeutic window extend?

\section{Can nonstroke acute hyperglycemia correction clinical trials be extrapolated to stroke?}

Randomized trials have studied aggressive correction of hyperglycemia in multiple acute illnesses, with varied results. The most convincing positive trial was the surgical intensive care unit (SICU) trial, in which postsurgical ventilated patients were randomized to aggressive or standard correction of hyperglycemia while in the SICU [18, Class I]. Patients treated aggressively had considerably lower mean glucose levels (by about $50 \mathrm{mg} / \mathrm{dL}$ ) and better clinical outcomes than patients under standard care. Significant benefits associated with aggressive hyperglycemia correction included decreased mortality, shorter time on ventilator, fewer bloodstream infections, and less critical-illness polyneuropathy. A similar trial with patients in the medical intensive care unit (MICU) showed less convincing results, without a statistically significant difference in the primary outcome (mortality) [19, Class I]. However, mortality among patients who stayed in the MICU for at least 3 days (secondary outcome) was significantly reduced in the aggressive insulin treatment group. The mean blood glucose difference between the treatment groups in that trial was about $51 \mathrm{mg} / \mathrm{dL}$.

In the latest ICU trial of hyperglycemia correction, 6104 patients were randomized to intensive or standard treatment for three or more days [20, Class I]. The mean blood glucose was about $35 \mathrm{mg} / \mathrm{dL}$ lower in the intensive group than in the standard group. The primary outcome, death, was more common in the intensive treatment group $(27.5 \%)$ than in the standard treatment group $(24.9 \% ; P=0.02)$. This treatment effect was similar among medical and surgical ICU patients. Severe hypoglycemia $(<40 \mathrm{mg} / \mathrm{dL})$ occurred in $6.8 \%$ of patients in the intensive group and $0.5 \%$ of those in the standard group.

One trial tested the efficacy of aggressive hyperglycemia correction in patients with sepsis [21]. The mean glucose levels were $39 \mathrm{mg} / \mathrm{dL}$ lower in the aggressive-treatment group than in the usual-care group. That trial was stopped early because of safety concerns about excess severe hypoglycemia $(<40 \mathrm{mg} / \mathrm{dL})$ in the aggressive-treatment group $(17.0 \%$ vs $4.1 \%$ in the control group). The severe hypoglycemia in the aggressively treated group was associated with a significantly higher rate of serious adverse events $(10.9 \%$ vs $5.2 \%$ in the control group).

Three randomized clinical trials of hyperglycemia correction in acute myocardial infarction have been reported [22-24]. Only the first trial showed significant benefit from aggressive insulin treatment [23]. In that trial, the mean glucose levels were $38 \mathrm{mg} / \mathrm{dL}$ lower in the aggressive-treatment group than in the control group, and mortality at 1 year $(18.6 \%)$ was significantly reduced versus the control group (26.1\%). In the two later randomized trials, the mean glucose differences between the treatment groups were smaller $(12-16 \mathrm{mg} / \mathrm{dL})$, which may have contributed to a neutral (no benefit) result. 
Two randomized trials studied the effects of aggressive hyperglycemia correction during coronary artery bypass surgery $[25,26]$. The smaller trial $(N=$ 141 ), in patients with diabetes mellitus and with a mean blood glucose difference between the two treatment groups of $122 \mathrm{mg} / \mathrm{dL}$, showed a benefit related to the aggressive intervention [26]. However, the larger trial $(N=$ 400 ), in patients mostly without diabetes and with a mean glucose difference between the two treatment groups of $43 \mathrm{mg} / \mathrm{dL}$, did not show a benefit [25].

These nonstroke illnesses involve considerably different pathophysiologic processes than acute brain ischemia and therefore are not comparable to stroke. The effects of hyperglycemia on acutely ischemic myocardium are likely to be much different than its effects on acutely ischemic brain cells. Also, much of the benefit from aggressive hyperglycemia reduction in the SICU trial could be attributed to reductions in critical-illness polyneuropathy and bloodstream infections, which are not applicable to most patients with acute stroke.

\section{Observational acute stroke hyperglycemia studies}

Human studies have largely been observational, and most of them show an association between hyperglycemia and inferior clinical outcomes from acute ischemic stroke $[10,27 \bullet, 28,29]$. The higher the glucose level during acute ischemic stroke, the worse the outcomes are. Some studies suggest a linear decrease in favorable outcomes as blood glucose increases [30,31]. One study found that during the initial $48 \mathrm{~h}$ of acute ischemic stroke, maximum glucose of $155 \mathrm{mg} / \mathrm{dL}$ was the best cutoff for predicting outcome [32]. In that study, however, the area under the receiver operating characteristic curve was only 0.66 (closer to 0.5 than to 1.0), indicating that this is not a distinct cutoff. Some findings suggest that patients with acute lacunar strokes (small subcortical infarcts caused by occlusion of small penetrating arterioles) have better outcomes with hyperglycemia $[30,33 \bullet]$. This result may be important and deserves further study.

One study analyzed the relation between acute hyperglycemia and recanalization in 139 patients with MCA occlusion treated with intravenous tissuetype plasminogen activator (tPA) [34]. The MCA occlusion was documented and followed with transcranial Doppler. After adjusting for stroke etiology and risk factors, poor recanalization was associated with admission hyperglycemia $(>158 \mathrm{mg} / \mathrm{dL}, P=0.03)$, proximal MCA occlusion $(P=0.03)$, and platelet count greater than $219,000 / \mathrm{mL}(P=0.03)$. However, chronic hyperglycemia determined by hemoglobin $\mathrm{A} 1 \mathrm{C}$ and fructosamine had no relation to recanalization. The investigators attributed the decreased recanalization associated with hyperglycemia to antifibrinolytic and hypercoagulable effects of hyperglycemia. The favored explanation was that glycation of the regulatory protein annexin II increases the formation of plasminogen/tPA/ annexin complexes, which interfere with fibrinolysis. The investigators also considered that hyperglycemia increases plasminogen activator-inhibitor concentrations.

We studied the relation between admission hyperglycemia and recanalization in 53 consecutive patients with proximal MCA occlusion treated with endovascular procedures by the University of California at Los Angeles 
(UCLA) Stroke Service between 2004 and 2009. Recanalization was classified by the Thrombolysis In Myocardial Infarction (TIMI) method: poor (no or incomplete poststenotic arterial filling, TIMI 0-1) or adequate (slow, but complete, or normal poststenotic arterial filling, TIMI 2-3) using serial magnetic resonance arteriograms. Patients with poor recanalization had somewhat higher admission blood glucose ( $154 \mathrm{vs} 139 \mathrm{mg} / \mathrm{dL} ; P=0.66$, Mann-Whitney test) and a higher rate of hyperglycemia (>140 mg/dL) (44\% vs $23 \%$; $P=0.10$, chi-square test) than patients with adequate recanalization. Our findings are consistent with the previous similar studies; our sample size is likely too small to show statistical significance.

Another important observation is the association between hyperglycemia and hemorrhagic transformation of infarcts. Patients with higher admission glucose levels are more likely to have a hemorrhagic conversion of their infarct $[29,31,35,36]$. We studied the relation between hyperglycemia and serious hemorrhagic transformation of infarcts in the same 53 consecutive patients just discussed. Serious hemorrhagic transformation was determined by serial MRI as confluent parenchymal hemorrhage with some mass effect within $24 \mathrm{~h}$ after stroke onset. Patients with serious hemorrhagic transformation had somewhat higher admission glucose levels $(159$ versus $140 \mathrm{mg} / \mathrm{dL}$; $P=0.19$, Mann-Whitney test $)$ and a higher rate of hyperglycemia $(>140 \mathrm{mg} /$ dL) (50\% vs $24 \% ; P=0.09$, chi-square test) than patients without serious hemorrhagic transformation. This finding is also consistent with previously published observations in patients treated with thrombolysis, and this complication also has been reported in animal studies [37,38].

In addition to clinical outcomes, there is MRI evidence of ischemic stroke worsening with hyperglycemia [39-42]. Higher blood glucose levels have been associated with greater acute infarct growth compared with lower glucose levels, based on acute and delayed diffusion and perfusion MRI. However, one study found that in a small subgroup $(N=11)$ of acute stroke patients with persistent arterial occlusion, treatment of hyperglycemia with intravenous insulin was associated with greater infarct growth [43]. Additional studies are needed to clarify these interesting observations.

\section{How might hyperglycemia exacerbate ischemic brain injury?}

The reproducible associations reported between hyperglycemia and poorer outcomes suggest a potential causal relationship. Multiple physiologic mechanisms have been proposed for how hyperglycemia might exacerbate ischemic brain injury (Table 1). The relative importance of the various injuryexacerbating mechanisms has not been established.

Much attention has been devoted to the study of brain lactate accumulation and brain acidosis due to hyperglycemia, but lactate has not been shown to exacerbate acute ischemic brain injury in animal studies [27•] or in a pilot clinical stroke trial [43].

Another potential mechanism by which hyperglycemia may exacerbate ischemic brain injury occurs at the level of microcirculation. Hyperglycemia can induce a variety of detrimental changes in cerebral endothelial cells within hours [13]. Acute hyperglycemia can impair cerebrovascular autoregulation, resulting in deleterious reperfusion predisposing to hemorrhagic 
Table 1. Derangements exacerbated by hyperglycemia during brain ischemia in animal studies, clinical studies, or both ${ }^{\mathrm{a}}$

Brain acidosis and increased lactate

Cytotoxic brain edema

Hemorrhagic transformation of infarcts

Impaired thrombolysis

Blood-brain barrier disruption

Cytotoxicity, slowed calcium recovery

Impaired ATP/energy recovery

Accumulation of free radicals

Impaired vascular reactivity

Stimulated inflammatory reactions

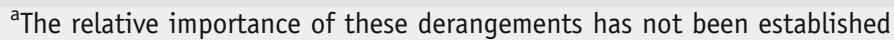

ATP-adenosine triphosphate

transformation of infarcts. Hyperglycemia can also stimulate inflammation through nuclear factor kappa-light-chain-enhancer of activated B cells (NF- $\kappa \mathrm{B})$, leading to upregulation of intracellular adhesion molecule 1, vascular cellular adhesion molecule 1, and E-selectin [13]. The adhesion molecules promote leukocyte adhesion to post-capillary venule walls, obstructing flow [44].

Hyperglycemia can also increase levels of plasminogen activator inhibitor 1 (PAI-1) by post-translational modification of proteins [13]. PAI-1 interferes with the actions of tPA, reducing the chance of early brain reperfusion. Another apparently important exacerbating mechanism of acute ischemic brain injury with hyperglycemia is the accumulation of free radicals. One study showed that glucose donates electrons to form superoxide radicals that exacerbate the injury during acute brain ischemia [45].

\section{Randomized clinical trials in acute stroke with hyperglycemia}

The supporting data outlined above have inspired five randomized pilot clinical trials of rapid hyperglycemia correction in acute ischemic stroke [43,4649]. These pilot trials have demonstrated the relative safety and feasibility of rapid hyperglycemia lowering with intravenous insulin as compared with subcutaneous insulin during acute stroke. These small pilot trials were not intended to show clinical efficacy of the intravenous insulin intervention.

One efficacy trial in acute stroke with hyperglycemia has been completed, the GIST-UK (Glucose Insulin Stroke Trial-United Kingdom) [50•, Class I]. In this trial, 933 acute stroke patients without previous insulin treatment were randomized within $24 \mathrm{~h}$ after stroke onset to infusions of normal saline or insulin for $24 \mathrm{~h}$. The mean admission glucose level was similar in the two treatment groups (137-141 mg/dL), and during treatment the difference in mean glucose between the two groups was only $10 \mathrm{mg} / \mathrm{dL}$. The only worrisome complication was an increase in mortality associated with the largest drops in blood glucose levels in the insulin-treated group. There were no significant differences in clinical outcomes, including mortality, neurologic def- 
icit, and handicap, between the two treatment groups. Although insulin infusion and hyperglycemia lowering may not be beneficial during acute stroke, additional potential reasons for the neutral results of the GIST-UK trial include delayed initiation of insulin infusion and the relatively small difference in glucose levels between the treatment groups.

Additional clinical efficacy trials are needed to address the uncertainty about the benefit of rapid hyperglycemia correction during acute stroke. These trials should differ from the GIST-UK trial by initiating the insulin infusion sooner and enrolling patients with higher glucose levels to achieve a greater difference in glucose between the treatment groups. If proven beneficial, a substantial proportion of acute stroke patients could be treated more effectively with a relatively inexpensive drug (insulin) that is familiar to health care providers. If this treatment proves ineffective, our efforts can be redirected toward other promising acute stroke therapies.

Another reason why additional randomized clinical trials are needed involves the costs of the treatment. Using intravenous insulin to rapidly control hyperglycemia (near $100 \mathrm{mg} / \mathrm{dL}$ ) during acute illness requires admission to a specialized hospital unit that is prepared to administer it-either an ICU or, in some hospitals, an intermediate unit (also termed a transitional, progressive, or stepdown unit). Consequently, safe intravenous insulin infusion therapy necessitates a greater personnel effort and eventually greater cost. To justify such additional effort, costs, and risk, we should await convincing evidence of efficacy from randomized clinical trials.

\section{Treatment}

\section{Lowering hyperglycemia during acute stroke}

Subcutaneous insulin

Subcutaneous insulin protocols usually can maintain blood glucose levels below $200 \mathrm{mg} / \mathrm{dL}$ in most patients, especially if basal insulin is added.

Subcutaneous regular insulin sliding scales are familiar to health care providers and seem safe because they typically lower blood glucose levels over a period of several hours and rarely result in hypoglycemia. The most simple sliding scales call for regular insulin doses based on the recent glucose levels.

More sophisticated treatment protocols include additional insulin doses for meals and long-acting basal insulin [51]. When available, an endocrinology consultant can optimize the acute treatment and help the transition to long-term care.

Daily insulin needs are likely to change rapidly during acute illness. The subcutaneous insulin sliding scales do not require admission to specialized hospital units or frequent glucose monitoring and appear to be the current standard of care in most clinical settings. However, it seems unreasonable that subcutaneous insulin treatment will rapidly 
and safely lower hyperglycemia to near $100 \mathrm{mg} / \mathrm{dL}$ and maintain such good control during acute stroke.

At this time, the only effective method to lower hyperglycemia to near $100 \mathrm{mg} / \mathrm{dL}$ within 4 to $5 \mathrm{~h}$ and to keep it near that level is to use intravenous insulin.

For ischemic stroke treated with thrombolytic drugs, it may be best to rapidly lower severe hyperglycemia. For example, if the admission blood glucose is approximately $300 \mathrm{mg} / \mathrm{dL}$ and the patient is a candidate for thrombolytic therapy, consider giving an intravenous bolus of regular insulin 8 units. Somewhat lower or higher insulin doses may be best for lesser or greater hyperglycemia. This bolus will start lowering the blood glucose in about $5 \mathrm{~min}$. A temporary continuous intravenous insulin infusion may then be used in most patients to maintain the glucose closer to normal levels (eg, below 180 or $140 \mathrm{mg} / \mathrm{dL}$ ).

There are multiple protocols that rapidly correct hyperglycemia in ICU patients. Some protocols have trade names $[52,53]$ and some do not [46]. Some academic institutions use protocols developed by local experts; some of these are available on their institutional websites, and many are publicly accessible on the Web [54]. Differences between these protocols that do not appear to be crucial at this time include whether to add potassium and/or a small amount of glucose to the solution. These protocols consider recent blood glucose levels but differ somewhat in the formulas used to determine the exact increases and decreases in insulin infusion rates over time. Such protocols have not been compared in head-to-head trials. They appear to have similar glucose-lowering efficiency and low risk of clinically significant hypoglycemia $(<40 \mathrm{mg} / \mathrm{dL})$. Computerized protocols are likely superior to paper-based protocols because they eliminate human error in calculating the infusion rate adjustments, they facilitate accurate data storage, and they have built-in reminders to recheck glucose levels.

Rapid and safe hyperglycemia correction requires glucose monitoring every 1 to $2 \mathrm{~h}$. Capillary, venous, or arterial glucose measurements differ somewhat in a consistent fashion over a large range of levels, but all are considered appropriate and reliable for monitoring. Transdermal and subcutaneous glucose sensing devices are also available, but they reflect the blood glucose with 15 to 30 min delay and thus should be supplemented with blood measurements when the blood glucose levels are dropping rapidly. Computerized programs for storing and tracking the glucose levels over time are ideal because they can show the results in a useful output rapidly.

\section{Acknowledgments}

We thank the following UCLA stroke investigators for their contribution to data collection: Jeffrey Saver, Sidney Starkman, Latisha Ali, Doojin Kim, Bruce Ovbiagele, Michael Froehler, Matthew Tenser, Monica Sapo, Noriko Salamon, Jignesh Gadhia, Farzad Buxey, Paul Vespa, Reza Jahan, Satoshi Tateshima, Nestor Gonzalez, Gary Duckwiler, and Fernando Vinuela. 
No potential conflicts of interest relevant to this article were reported.

\section{Open Access}

This article is distributed under the terms of the Creative Commons Attribution Noncommercial License which permits any noncommercial use, distribution, and reproduction in any medium, provided the original author(s) and source are credited.

\section{References and Recommended Reading}

Papers of particular interest, published recently, have been highlighted as:

- Of importance

$\bullet \quad$ Of major importance

1. Gentile NT, Seftchick MW, Huynh T, et al.: Decreased mortality by normalizing blood glucose after acute ischemic stroke. Acad Emerg Med 2006, 13:174-180.

2. Williams LS, Rotich J, Qi R, et al.: Effects of admission hyperglycemia on mortality and costs in acute ischemic stroke. Neurology 2002, 59:67-71.

3. Pencina MJ, D'Agostino Sr RB, Larson MG, et al.: Predicting the 30-year risk of cardiovascular disease: The Framingham Heart Study. Circulation 2009, 119:3078-3084.

4. Spencer EA, Pirie KL, Stevens RJ, et al.: Diabetes and modifiable risk factors for cardiovascular disease: The Prospective Million Women Study. Eur J Epidemiol 2008, 23:793-799.

5. Whisnant JP, Wiebers DO, O'Fallon WM, et al.: A population-based model of risk factors for ischemic stroke: Rochester, Minnesota. Neurology 1996, 47:1420-1428.

6. Johnston SC, Rothwell PM, Nguyen-Huynh MN, et al.: Validation and refinement of scores to predict very early stroke risk after transient ischaemic attack. Lancet 2007, 369:283-292.

7. Ray KK, Seshasai SR, Wijesuriya S, et al.: Effect of intensive control of glucose on cardiovascular outcomes and death in patients with diabetes mellitus: a meta-analysis of randomised controlled trials. Lancet 2009, 373:1765-1772.

8. Alberti KG, Zimmet P, Shaw J: The metabolic syndrome-a new worldwide definition. Lancet 2005, 366:1059-1062.

9. De Keyser J, Sulter G, Luiten PG: Clinical trials with neuroprotective drugs in acute ischaemic stroke: Are we doing the right thing? Trends Neurosci 1999, 22:535-540.
10. Bruno A, Williams LS, Kent TA: How important is hyperglycemia during acute brain infarction? Neurologist 2004, 10:195-200.

11. de Courten-Myers G, Myers RE, Schoolfield L: Hyperglycemia enlarges infarct size in cerebrovascular occlusion in cats. Stroke 1988, 19:623-630.

12. Kent TA, Soukup VM, Fabian RH: Heterogeneity affecting outcome from acute stroke therapy: making reperfusion worse. Stroke 2001, 32:2318-2327.

13. Martini SR, Kent TA: Hyperglycemia in acute ischemic stroke: a vascular perspective. J Cereb Blood Flow Metab 2007, 27:435-451.

14. Quast MJ, Wei J, Huang NC, et al.: Perfusion deficit parallels exacerbation of cerebral ischemia/ reperfusion injury in hyperglycemic rats. J Cereb Blood Flow Metab 1997, 17:553-559.

15. Bomont L, MacKenzie ET: Neuroprotection after focal cerebral ischaemia in hyperglycaemic and diabetic rats. Neurosci Lett 1995, 197:53-56.

16. Meden P, Andersen M, Overgaard K, et al.: The effects of early insulin treatment combined with thrombolysis in rat embolic stroke. Neurol Res 2002, 24:399-404.

17. Zhu CZ, Auer RN: Optimal blood glucose levels while using insulin to minimize the size of infarction in focal cerebral ischemia. J Neurosurg 2004, 101:664-668.

18. van den Berghe G, Wouters P, Weekers F, et al.: Intensive insulin therapy in the critically ill patients. N Engl J Med 2001, 345:1359-1367.

19. van den Berghe G, Wilmer A, Hermans G, et al.: Intensive insulin therapy in the medical ICU. $N$ Engl J Med 2006, 354:449-461. 
20. Finfer S, Chittock DR, Su SY, et al.: Intensive versus conventional glucose control in critically ill patients. N Engl J Med 2009, 360:1283-1297.

21. Brunkhorst FM, Engel C, Bloos F, et al.: Intensive insulin therapy and pentastarch resuscitation in severe sepsis. N Engl J Med 2008, 358:125-139.

22. Cheung NW, Wong VW, McLean M: The Hyperglycemia: Intensive Insulin Infusion In Infarction (HI-5) study: a randomized controlled trial of insulin infusion therapy for myocardial infarction. Diab Care 2006, 29:765-770.

23. Malmberg K, Ryden L, Efendic S, et al.: on behalf of the DIGAMI Study Group: Randomized trial of insulin-glucose infusion followed by subcutaneous insulin treatment in diabetic patients with acute myocardial infarction (DIGAMI study): effects on mortality at 1 year. J Am Coll Cardiol 1995, 26:57-65.

24. Malmberg K, Ryden $\mathrm{L}$, Wedel $\mathrm{H}$, et al.: Intense metabolic control by means of insulin in patients with diabetes mellitus and acute myocardial infarction (DIGAMI 2): effects on mortality and morbidity. Eur Heart J 2005, 26:650-661.

25. Gandhi GY, Nuttall GA, Abel MD, et al.: Intensive intraoperative insulin therapy versus conventional glucose management during cardiac surgery: a randomized trial. Ann Intern Med 2007, 146:233-243.

26. Lazar HL, Chipkin SR, Fitzgerald CA, et al.: Tight glycemic control in diabetic coronary artery bypass graft patients improves perioperative outcomes and decreases recurrent ischemic events. Circulation 2004, 109:1497-1502.

27. McCormick MT, Muir KW, Gray CS, Walters MR Management of hyperglycemia in acute stroke: how, when, and for whom? Stroke 2008, 39:21772185.

This article presents a thorough overview of the published findings in animal and clinical studies of hyperglycemia in acute ischemic stroke.

28. Poppe AY, Majumdar SR, Jeerakathil T, et al.: Admission hyperglycemia predicts a worse outcome in stroke patients treated with intravenous thrombolysis. Diab Care 2009, 32:617-622.

29. Yong M, Kaste M: Dynamic of hyperglycemia as a predictor of stroke outcome in the ECASS-II trial. Stroke 2008, 39:2749-2755.

30. Bruno A, Biller J, Adams Jr HP: et al.: Acute blood glucose level and outcome from ischemic stroke. Trial of Org 10172 in Acute Stroke Treatment (TOAST) investigators. Neurology 1999, 52:280-284.

31. Bruno A, Levine SR, Frankel MR, et al.: Admission glucose level and clinical outcomes in the NINDS rt-PA Stroke Trial. Neurology 2002, 59:669-674.

32. Fuentes B, Castillo J, SanJose B, et al.: The prognostic value of capillary glucose levels in acute stroke: The Glycemia In Acute Stroke (GLIAS) study. Stroke 2009, 40:562-568.
33. Uyttenboogaart M, Koch MW, Stewart RE, et al.: Moderate hyperglycaemia is associated with favourable outcome in acute lacunar stroke. Brain 2007, 130:1626-1630.

This article discusses a potentially important, previously reported observation - that hyperglycemia during acute ischemic stroke may somehow be advantageous in some patients, such as those with lacunar stroke subtype.

34. Ribo M, Molina C, Montaner J, et al.: Acute hyperglycemia state is associated with lower tPA-induced recanalization rates in stroke patients. Stroke 2005, 36:1705-1709.

35. Demchuk AM, Morgenstern LB, Krieger DW, et al.: Serum glucose level and diabetes predict tissue plasminogen activator-related intracerebral hemorrhage in acute ischemic stroke. Stroke 1999, 30:34-39.

36. Kase CS, Furlan AJ, Wechsler LR, et al.: Cerebral hemorrhage after intra-arterial thrombolysis for ischemic stroke: the PROACT II trial. Neurology 2001, 57:1603-1610.

37. de Courten-Myers GM, Kleinholz M, Holm P, et al.: Hemorrhagic infarct conversion in experimental stroke. Ann Emerg Med 1992, 21:120-126.

38. Ergul A, Elgebaly MM, Middlemore ML, et al.: Increased hemorrhagic transformation and altered infarct size and localization after experimental stroke in a rat model type 2 diabetes. $B M C$ Neurol 2007, 7:33.

39. Baird TA, Parsons MW, Phanh T, et al.: Persistent poststroke hyperglycemia is independently associated with infarct expansion and worse clinical outcome. Stroke 2003, 34:2208-2214.

40. Els T, Klisch J, Orszagh $\mathrm{M}$, et al.: Hyperglycemia in patients with focal cerebral ischemia after intravenous thrombolysis: influence on clinical outcome and infarct size. Cerebrovasc Dis 2002, 13:89-94.

41. Parsons MW, Barber PA, Desmond PM, et al.: Acute hyperglycemia adversely affects stroke outcome: a magnetic resonance imaging and spectroscopy study. Ann Neurol 2002, 52:20-28.

42. Ribo M, Molina CA, Delgado P, et al.: Hyperglycemia during ischemia rapidly accelerates brain damage in stroke patients treated with tPA. J Cereb Blood Flow Metab 2007, 27:1616-1622.

43. McCormick M, Hadley D, McLean JR, et al.: Randomized, controlled trial of insulin for acute poststroke hyperglycemia. Ann Neurol 2010, 67:570-578.

44. del Zoppo GJ, Mabuchi T: Cerebral microvessel responses to focal ischemia. J Cereb Blood Flow Metab 2003, 23:879-894.

45. Suh SW, Shin BS, Ma H, et al.: Glucose and NADPH oxidase drive neuronal superoxide formation in stroke. Ann Neurol 2008, 64:654-663. 
46. Bruno A, Kent TA, Coull BM, et al.: Treatment of hyperglycemia in ischemic stroke (THIS): a randomized pilot trial. Stroke 2008, 39:384-389.

47. Johnston KC, Hall CE, Kissela BM, et al.: Glucose Regulation in Acute Stroke Patients (GRASP) trial: a randomized pilot trial. Stroke 2009, 40: 3804-3809.

48. Kreisel SH, Berschin UM, Hammes HP, et al.: Pragmatic management of hyperglycaemia in acute ischaemic stroke: safety and feasibility of intensive intravenous insulin treatment. Cerebrovasc Dis 2009 27:167-175.

49. Walters MR, Weir CJ, Lees KR: A randomised, controlled pilot study to investigate the potential benefit of intervention with insulin in hyperglycaemic acute ischaemic stroke patients. Cerebrovasc Dis 2006, 22:116-122.

50. Gray CS, Hildreth AJ, Sandercock PA, et al.: Glucosepotassium-insulin infusions in the management of post-stroke hyperglycaemia: The UK Glucose Insulin in Stroke Trial (GIST-UK). Lancet Neurol 2007, 6:397-406.
This was the first clinical efficacy trial of strict hyperglycemia correction with intravenous insulin versus standard care in acute ischemic stroke. No efficacy was detected, possibly because the difference in glucose levels between the two treatment groups was relatively small.

51. Moghissi E: Hospital management of diabetes: beyond the sliding scale. Cleve Clin J Med 2004, 71:801-808.

52. Davidson PC, Steed RD, Bode BW, et al.: Glucommander: a computer-directed intravenous insulin system shown to be safe, simple, and effective in 120, $618 \mathrm{~h}$ of operation. Diab Care 2005, 28:2418-2423.

53. Juneja R, Roudebush C, Kumar N, et al.: Utilization of a computerized intravenous insulin infusion program to control blood glucose in the intensive care unit. Diabetes Technol Ther 2007, 9:232-240.

54. Glycemic Control Resource Room Project Team: Insulin orders \& protocols: IV insulin infusions. Available at www.Hospitalmedicine.Org/resourcer oomredesign/html/12clinical_tools/04_insulin_ ordersiv.Cfm. Accessed July 23, 2010. 